\title{
Smartphones and Hearing Loss: There's an App for That!
}

\author{
Lori Zitelli, Au.D. ${ }^{1,2}$ and Elaine Mormer, Ph.D. ${ }^{1}$
}

\section{ABSTRACT}

Smartphone use has become increasingly prevalent and patients are frequently using this technology to engage in health-related activities. There are a variety of smartphone applications that can be used to assist individuals with hearing loss, particularly during extreme situations such as a global pandemic which results in restricted face-toface interactions. The hearing-related applications can be divided into four categories: (1) screening and assessment, (2) intervention and rehabilitation, (3) education and information, and (4) assistive tools. Several subcategories of applications in the assistive tools category are described that may be of interest to individuals with hearing loss, whether they wear hearing aids or not. Examples of the apps along with links are included for each subcategory.

KEYWORDS: smartphone, applications, hearing loss

The era of electronic health (e-health) began in the late 1990s. While the specific definition of this term has evolved over time, it has grown to represent healthcare provided on an electronic platform that is evidence based, efficient, empowering, and easy to use. ${ }^{1}$ With the restricted access to healthcare imposed by a pandemic, there has been an increased interest in the availability of mobile health (m-health) services-care that is accessed via mobile devices such as smartphones or tablets-to address patients' health-related needs. ${ }^{2}$ The Pew Research Center estimates that $96 \%$ of American adults own a cell phone, with $81 \%$ reporting that they own a smartphone. ${ }^{3}$ Additionally, children ${ }^{4}$ and elderly adults ${ }^{3}$ often can overcome common barriers (physical limitations, lack of knowledge, etc.) to utilizing this technology. ${ }^{5}$

The field of audiology had already embraced this trend prior to 2020 and, as a result, m-health solutions for communication challenges have become pervasive in clinical settings. Many audiologists have assimilated smartphone technology into their rehabilitation plans and, despite some frustration related to Bluetooth connectivity, ${ }^{6}$ have demonstrated a willingness to provide $\mathrm{m}$-health services to their patients. ${ }^{7}$
${ }^{1}$ Department of Communication Science and Disorders, School of Health and Rehabilitation Sciences, University of Pittsburgh; ${ }^{2}$ UPMC, Pittsburgh, Pennsylvania.

Address for correspondence: Lori Zitelli, Au.D., Department of Audiology, UPMC Eye and Ear Institute, 203 Lothrop Street, 4th Floor, Pittsburgh, PA 15213 (e-mail: Zitellild@upmc.edu).
Wireless Connectivity for People with Hearing Loss; Guest Editor, Linda M. Thibodeau, Ph.D.

Semin Hear 2020;41:266-276. Copyright (C) 2020 by Thieme Medical Publishers, Inc., 333 Seventh Avenue, New York, NY 10001, USA. Tel: +1(212) 760-0888.

DOI: https://doi.org/10.1055/s-0040-1718712.

ISSN 0734-0451. 
The use of applications (apps) goes hand in hand with smartphone use. Of note, hundreds of apps that are specific to hearing have been identified and sorted into four categories ${ }^{8}$ :

1. Screening and assessment.

2. Intervention and rehabilitation.

3. Education and information.

4. Assistive tools.

The goal of this article is to describe several subgroups of smartphone apps falling into the assistive tools category above. This list is by no means exhaustive-rather, it is designed to give examples of apps that are available for purposes related to hearing or hearing loss and provide the necessary resources to access them. The best solution for any given communication challenge is the one that works best for each individual user. Sometimes this solution involves hearing aids. Sometimes, as described later, it does not. When treatment options are limited by financial concerns, it could be argued that, given the myriad of $m$-health solutions that are readily available, an individual with hearing loss may be better served by purchasing a smartphone and data plan than hearing aids. There are many apps which were not developed specifically for individuals with hearing loss or tinnitus, but can be used by people with auditory issues to enhance communication, create alerting options, distract attention from tinnitus, etc.

In the context of the COVID-19 pandemic, apps that provide live captioning options to patients with hearing loss are more important than ever. The ability to convert speech to text can be useful in many situations. Fortunately, there are several options to provide this option for patients (some of which will be discussed later in this document). Patients and providers are both likely to be wearing masks that obscure facial cues and make lipreading impossible while communicating in the office. Fig. 1 outlines some suggestions for optimizing communication while wearing a mask. Telemedicine video and phone visits will be attractive options for patients who would prefer to be evaluated or receive care at a distance, and individuals with hearing loss may need captioning assistance to communicate successfully using these mediums. Additionally, many of our patients will be practicing physical distancing and forced to rely on telecommunications to stay socially connected to family and friends.

The remainder of this article will focus on select examples of smartphone apps in the assistive tools category in each of the following subcategories for both the Apple operating system (iOS) (Apple) and Android platforms (Fig. 2).

Please note that this technology is continually evolving and, although all links were active at the time of publication, the information may become outdated over time. Each subcategory will include a chart which includes the application's name, the platform that supports it, the cost, a quick response (QR) code, and any relevant notes. The $\mathrm{QR}$ code can be scanned with a smartphone camera and the link to download the app will open in the smartphone browser. Although some apps included in this list may be capable of working offline (i.e., without a $\mathrm{Wi}-\mathrm{Fi}$ or data plan connection), many of the apps listed below require an internet connection. Additionally, this connection would be required for all users involved if internet-based communication is occurring through the app.

\section{SOUND AMPLIFIER APPS}

The subcategory of amplifiers includes examples of apps that utilize a microphone of the listener's choice (smartphone, headset mic, and external mic) to pick up sounds, amplify sounds to a preferred level, and deliver them to the listener through headphones or ear buds. Though, like personal sound amplification products (PSAPs), these apps are not specifically intended to compensate for hearing loss, listeners may find them useful for improving the perception of a desired signal in various listening conditions (Table 1).

\section{FACE-TO-FACE COMIMUNICATION (VIDEO CALL) APPS (WITHOUT CAPTIONING)}

Apps designed to facilitate face-to-face communication (i.e., video chat) involve the use of both audio and video signals. Visual cues can enhance understanding of individuals who have hearing loss. Users must grant these apps permission to utilize their smartphone's microphone and camera(s) (Table 2). 


\section{Wearing A Mask while Communicating with Individuals who have Hearing Loss}
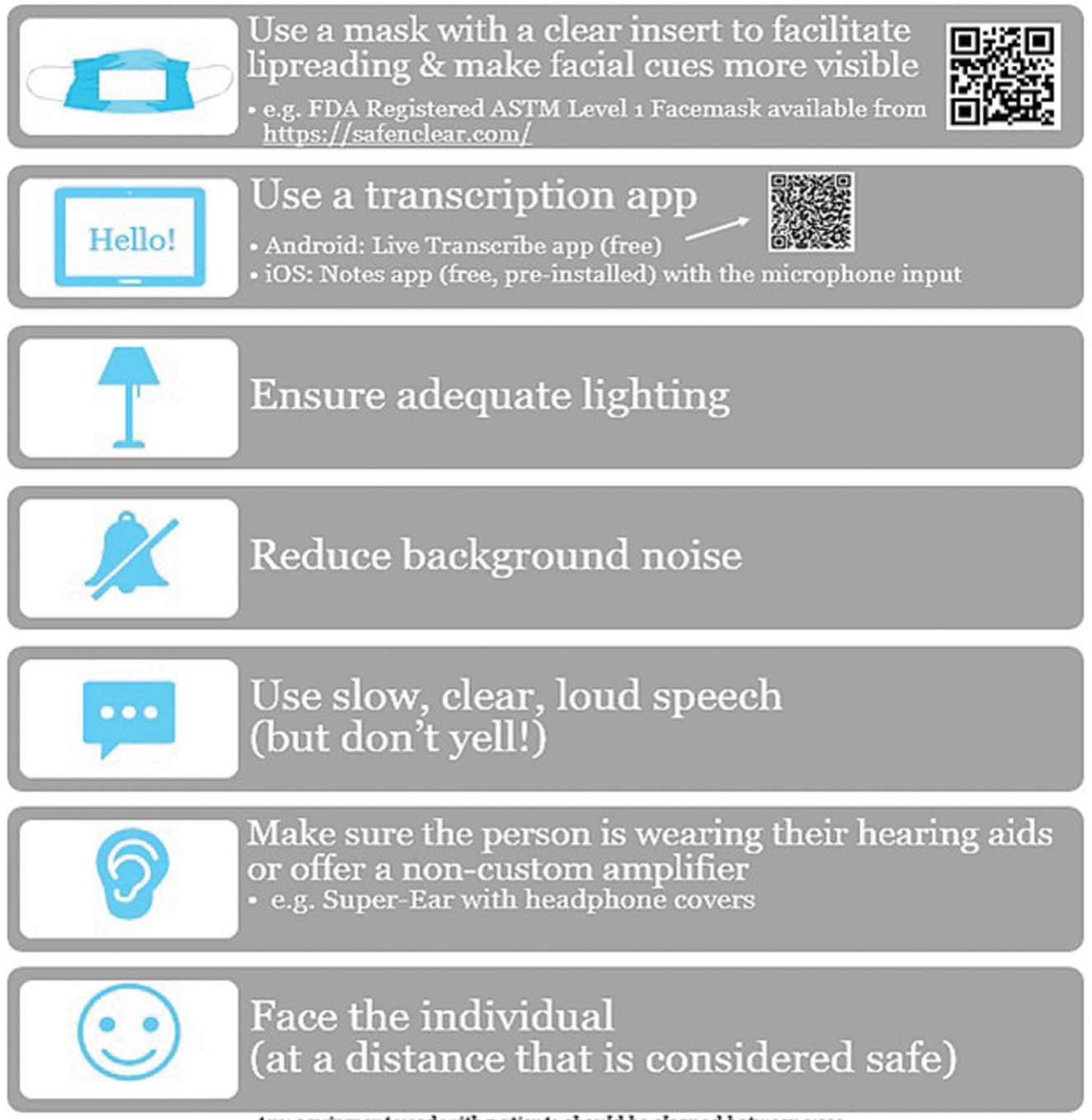

Any equipment used with patients should be cleaned between uses.

Figure 1 Wearing a mask while communicating with individuals who have hearing loss.

\section{PHONE AND VIDEO CALL APPS (WITH CAPTIONING)}

Other phone and video call apps used to facilitate social connections do so with the addition of captioning, which increases accessibility for individuals who have hearing loss. Landline phones with captioning abilities are available, but smartphone users can utilize apps designed for this purpose to achieve a similar outcome (Table 3).

\section{LIVE COMMUNICATION CAPTIONING APPS}

Live communication captioning is likely to be of interest to patients with hearing loss and providers alike. A variety of options are available which will allow individuals with hearing loss to follow conversations by reading live transcriptions of the interactions (Table 4). 

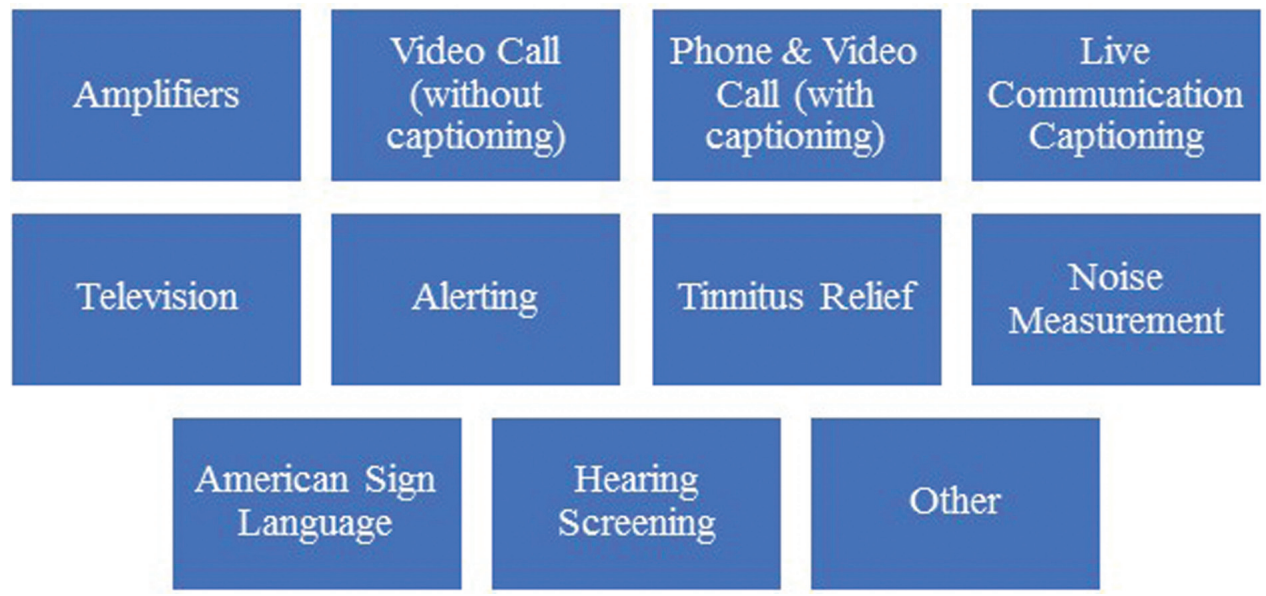

Figure 2 Subcategories of apps in "Assistive Tools" category.

Table 1 Sound Amplifier Apps

\begin{tabular}{lll}
\hline Name & Mobile Ears & Super Ear Tool \\
\hline Platform & iOS only & Android only \\
Cost & Free & Free \\
OR Code & a &
\end{tabular}

Notes

This app must be used with headphones plugged in; requires $\mathrm{iOS} 9.3$ or later

Can be used with Bluetooth headphones; requires Android 4.2 or later

\section{TELEVISION APPS}

In crowded environments (e.g., airports, restaurants, sports bars, and gyms), hearing the television can be difficult if not impossible. The apps in this subcategory help users to improve signal-to-noise ratio when listening to television audio or view subtitles on their smartphone. Some smartphones provide the ability to activate captions for television programs directly through the phone. This capability would be activated under the "Accessibility" menu under the phone settings (Table 5).

\section{ALERTING APPS}

Individuals with hearing loss often rely on alerting devices (e.g., vibration, flashing lights, and amplified sounds) to warn them when their doorbell, smoke alarm, or alarm clock has been activated or when their phone is ringing. The apps listed in Table 6 can turn a smartphone into an alerting device that will accomplish some of these goals.

\section{TINNITUS RELIEF APPS}

Many individuals who experience or suffer from tinnitus find that sound enrichment is beneficial (especially in very quiet environments) to distract them from their tinnitus. A few apps that use sound to facilitate relaxation or reduced awareness of tinnitus are listed in Table 7.

\section{NOISE MEASUREMENT APPS}

Individuals who are concerned about damaging their hearing or exacerbating their tinnitus may 
Table 2 Video Call (without Captioning) Apps

\begin{tabular}{lll}
\hline Name & FaceTime & WhatsApp Messenger \\
\hline Platform & iOS only & iOS and Android \\
Cost & Free & \\
QR Code & &
\end{tabular}

QR Code
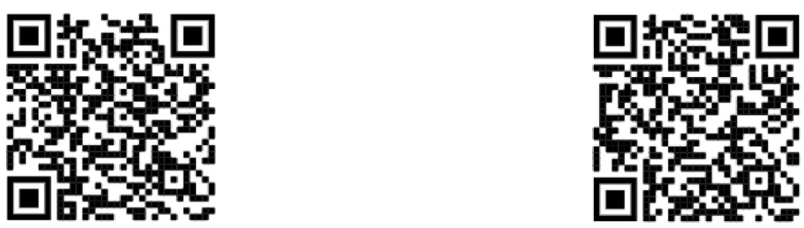

(iOS)

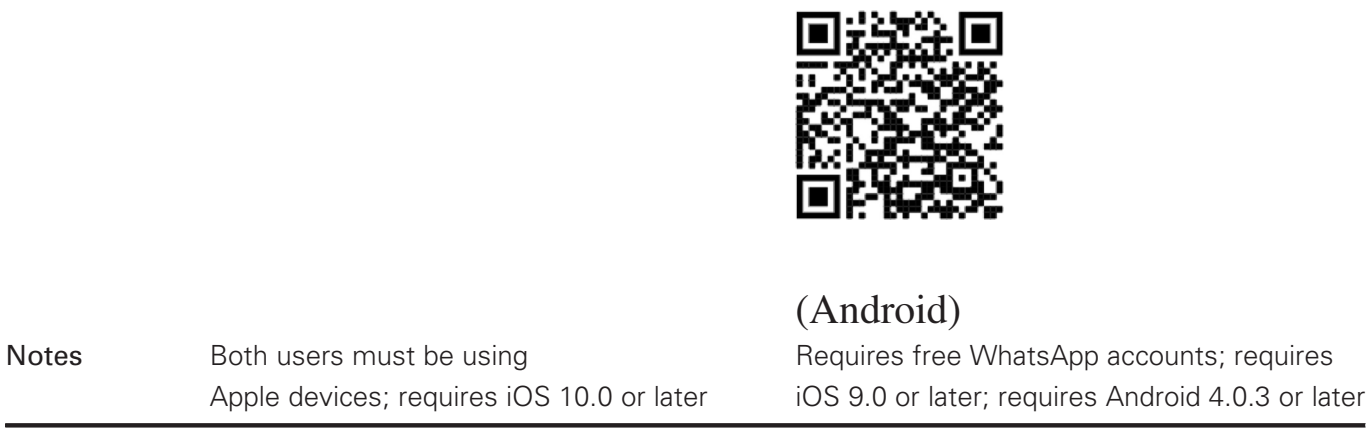

be interested in calculating their noise dose. Apps that measure sound levels using the smartphone microphone or use crowdsourced reporting can provide an estimate of noise exposure in any given place. Note that smartphone-based measurements may not comply with calibration standards required of approved sound level meters (Table 8).

\section{AMERICAN SIGN LANGUAGE APPS}

The deaf community in our country is composed of individuals who communicate primarily through American Sign Language. The app listed in Table 9 includes demonstrations of the signs for numbers, letters, commonly used phrases, and basic signs that may help someone who does not know any signs to convey rudimentary ideas using American Sign Language.

\section{HEARING SCREENING APPS}

Because hearing screenings often lead to comprehensive hearing evaluations when individuals do not pass, hearing screening apps have become popular. Individuals can use the apps listed in Table 10 to complete a digits-in-noise screening and connect to an audiologist for further care. Please scan the QR codes in Table 10 to read the disclaimers associated with each hearing screening app.

\section{OTHER APPS}

There are likely other apps available that would be of interest to individuals with hearing loss who do not fall into any of the specific categories mentioned earlier. Google Maps is one such app. It is primarily a location services app that provides navigation, but the app also provides attendance patterns for public places for each day of the week. Someone with hearing loss may be eager to use this feature to determine the quietest day and time to meet a friend for coffee at a local coffee shop (the time of day that is typically the least busy in that establishment; Table 11). 


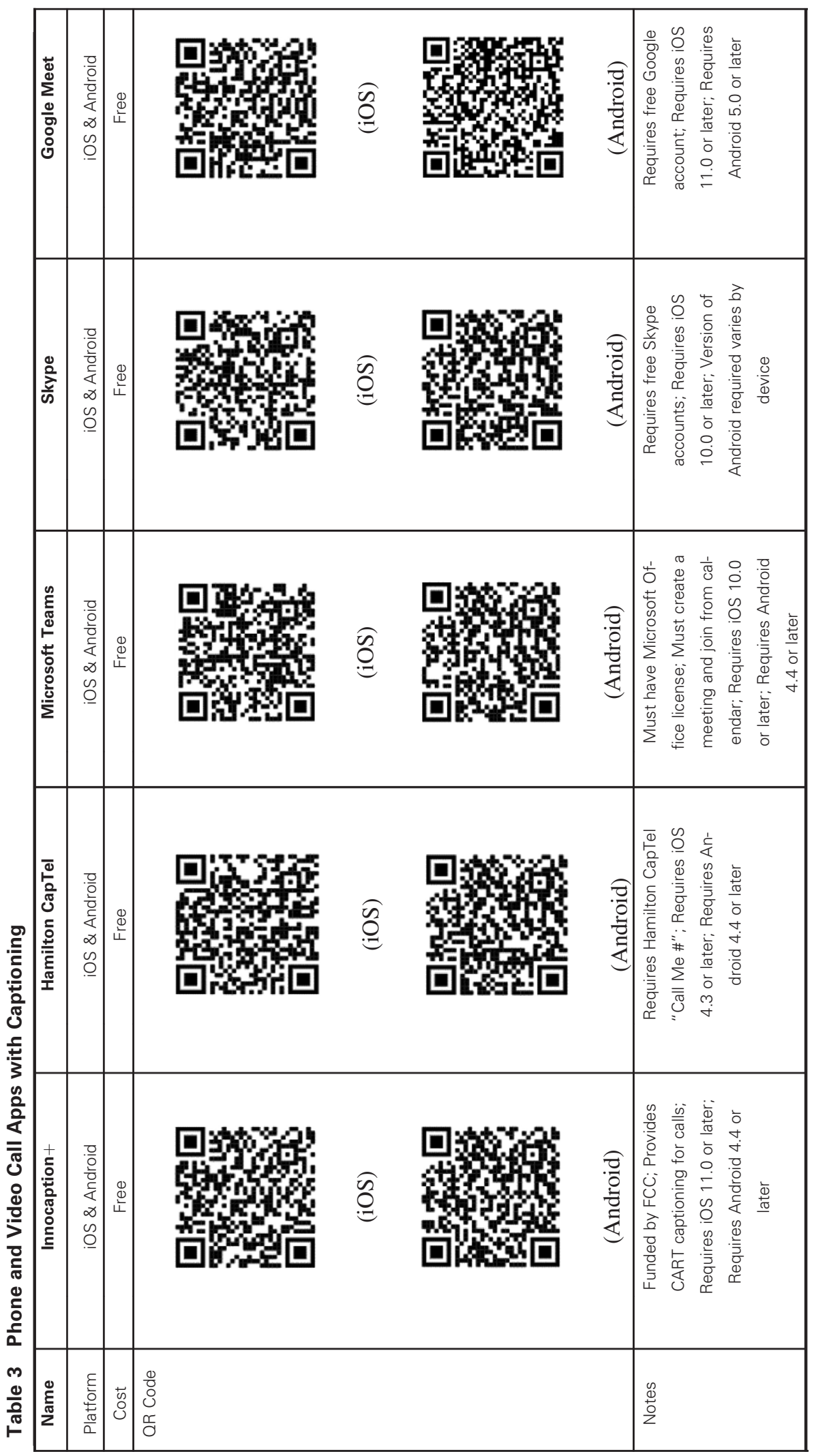




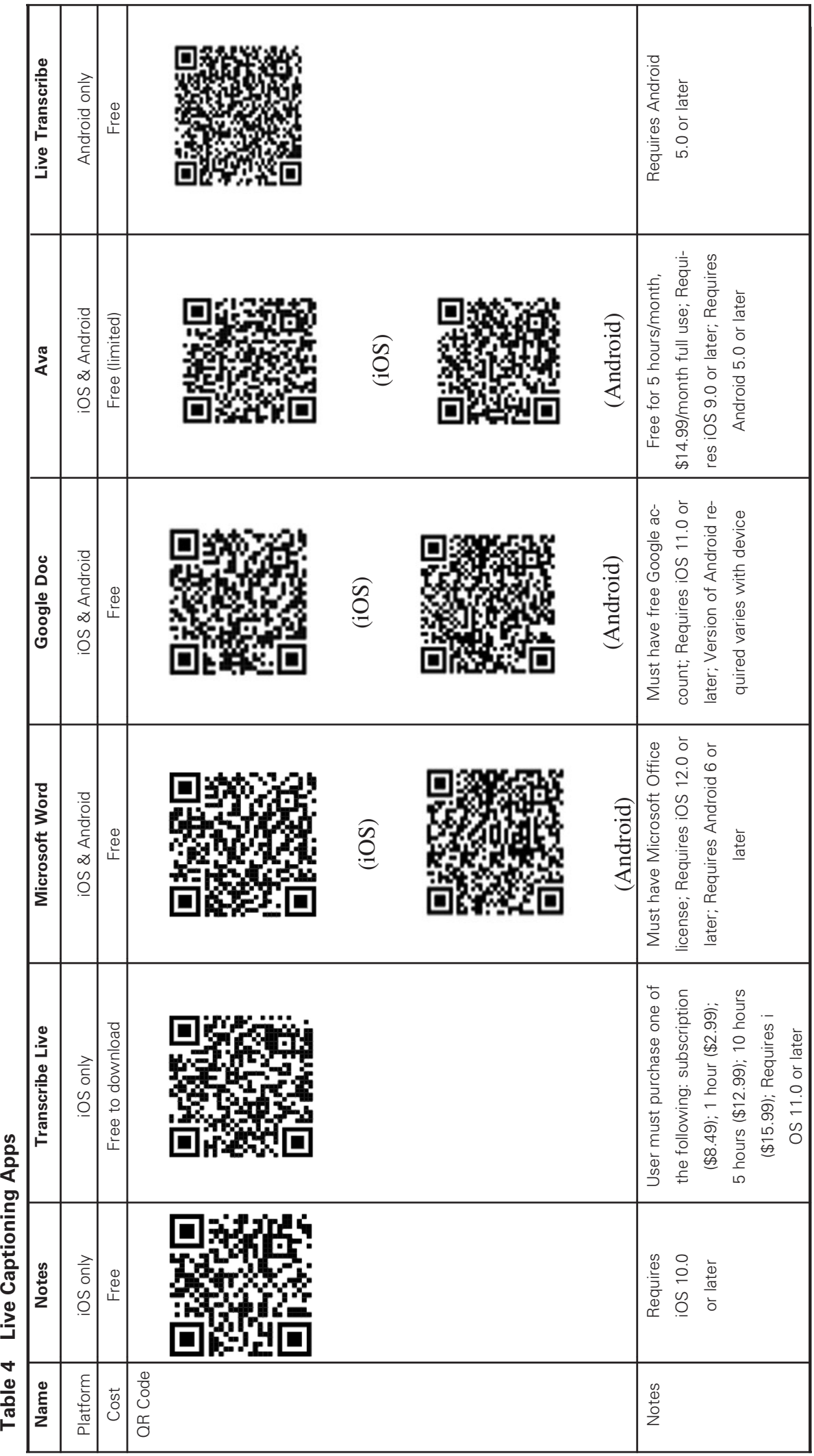


Table 5 Television Apps

\begin{tabular}{lll}
\hline Same & Subtitles Viewer & OOS only \\
Flatform & Free limited) \\
OR Code &
\end{tabular}

Table 6 Alerting Apps

\begin{tabular}{llll}
\hline Name & Alarm clock: missions, tasks & Sound alert & Flash alarm clock \\
\hline Platform ${ }^{\circ}$ OS only & iOS and Android & Android only \\
Cost $\quad$ Free &
\end{tabular}

(iOS)

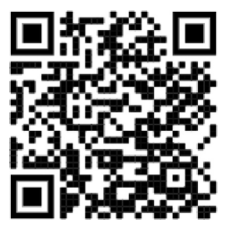

(Android) 
Table 7 Tinnitus Relief Apps

\begin{tabular}{lll}
\hline Name & ReSound relief & iOS and Android \\
Platform & Fost \\
OR Code &
\end{tabular}

Table 8 Noise Measurement Apps

\begin{tabular}{lll}
\hline Name & Decibel X & SoundPrint \\
Platform & IOS and Android \\
OR Code & Free
\end{tabular}


Table 9 American Sign Language Apps

\begin{tabular}{ll}
\hline Name & American Sign Langus \\
Platform \\
OR Code
\end{tabular}

\section{(Android)}

Table 10 Hearing Screening Apps

\begin{tabular}{lll}
\hline Name & hearScreen USA & hearWHO \\
\hline Platform & iOS and Android & iOS and Android \\
Cost & Free & Free \\
OR Code & &
\end{tabular}

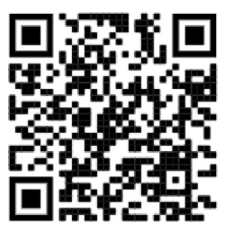

(iOS)

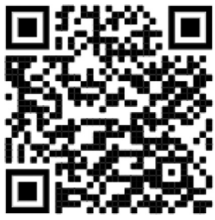

(Android)

Notes

Developed in collaboration with the American Academy of Audiology; requires iOS 10.0 or later; requires Android 5.0 or later

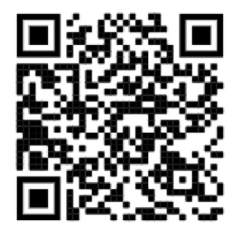

$(\mathrm{iOS})$

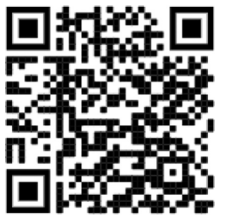

(Android)

Provided by the World Health Organization; requires iOS 11.0 or later; requires Android 5.0 or later 
Table 11 Other Apps

\begin{tabular}{ll}
\hline Name & Google Maps \\
\hline Platform & iOS and Android \\
Cost & Free \\
QR Code &
\end{tabular}

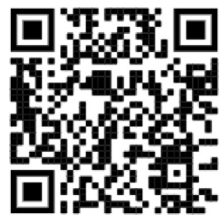

(iOS)

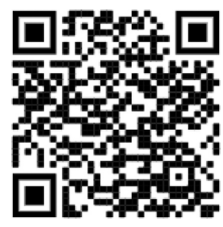

\section{(Android)}

Notes $\quad$ Requires iOS 11.0 or later; version of Android required varies with device

\section{CONCLUSION}

As smart devices continue to permeate Americans' personal and professional lives, healthcare providers will be required to adapt and integrate this technology into their evaluations and treatment plans. This will likely be the case even when healthcare returns to "pre-pandemic" operations. Audiologists should be aware of the types of hearing-related offerings in the iOS and Android app stores to support their patients who are unable to pursue customized amplification or who choose not to follow that specific pathway of hearing care. For certain

patients, these apps may provide a sufficient solution to their hearing-related problems while allowing them to achieve some success when normal communication interactions are not possible as the result of unforeseen challenges such as the global pandemic.

\section{CONFLICT OF INTEREST}

None declared.

\section{REFERENCES}

1. Eysenbach G. What is e-health? J Med Internet Res 2001;3(02):E20

2. Free C, Phillips G, Felix L, Galli L, Patel V, Edwards P. The effectiveness of M-health technologies for improving health and health services: a systematic review protocol. BMC Res Notes 2010;3 (01):250

3. PEW Research Center. Demographics of Mobile Device Ownership and Adoption in the United States. Available at: https://www.pewresearch.org/ internet/fact-sheet/mobile/. Accessed June 2, 2020

4. Yadav S, Chakraborty P. Children aged two to four are able to scribble and draw using a smartphone app. Acta Paediatr 2017;106(06):991-994

5. Mohadisdudis $\mathrm{H}$, Ali N. A study of smartphone usage and barriers among the elderly. 3rd International Conference on User Science and Engineering. September 2-5, 2014:109-114

6. Ng SL, Phelan S, Leonard M, Galster J. A qualitative case study of smartphone-connected hearing aids: influences on patients, clinicians, and patientclinician interactions. J Am Acad Audiol 2017;28 (06):506-521

7. Kimball SH, Singh G, John AB, Jenstad LM. Implications and attitudes of audiologists towards smartphone integration in hearing healthcare. Hear Res 2018;369:15-23

8. Paglialonga A, Tognola G, Pinciroli F. Apps for hearing science and care. Am J Audiol 2015;24(03): 293-298 